“C 2019 IEEE. Personal use of this material is permitted. Permission from IEEE must be obtained for all other uses, in any current or future media, including reprinting/republishing this material for advertising or promotional purposes, creating new collective works, for resale or redistribution to servers or lists, or reuse of any copyrighted component of this work in other works." 


\title{
Joint Communication and Radar Sensing in 5G Mobile Network by Compressive Sensing
}

\author{
Md Lushanur Rahman ${ }^{1}$, Peng-fei Cui ${ }^{1}$, J. Andrew Zhang ${ }^{1}$, Xiaojing Huang ${ }^{1}$, Y. Jay Guo ${ }^{1}$ and Zhiping Lu$^{2}$ \\ ${ }^{1}$ University of Technology Sydney (UTS), Global Big Data Technologies Centre (GBDTC), Australia \\ ${ }^{2}$ China Academy of Telecommunication Technology (CATT), Beijing, China \\ \{MdLushanur.Rahman; pengfei.cui\}@ student.uts.edu.au; \\ \{Andrew.Zhang; Xiaojing.Huang; Jay.Guo\}@uts.edu.au; luzhiping@catt.cn.
}

\begin{abstract}
There is growing interest in integrating communication and radar sensing into one system. However, very limited results are reported on how to realize sensing using complicated mobile signals when joint communication and radar sensing (JCAS) is applied to mobile networks. This paper studies radar sensing using one-dimension (1D) to 3D compressive sensing techniques, referring to signals compatible with latest fifth generation (5G) new radio (NR) standard. We demonstrate that radio sensing using both downlink and uplink $5 \mathrm{G}$ signals can be realized with reasonable performance using these CS techniques, and highlight the respective advantages and disadvantages of these techniques. 1

Keywords-Joint Communication and Radar Sensing, Compressive Sensing (CS), Clustered multipath and 5G mobile network.
\end{abstract}

\section{INTRODUCTION}

The recently proposed perceptive mobile network [1], [2], with a conceptual plot provided in Fig. 1, can provide integrated communication and radio sensing in one system. On a unified sensing platform, information associated with e.g., human behaviour, moving objects and environmental changes can be extracted from communication signals via sensing parameters. Estimation of sensing parameters, such as the delay, angle of arrival (AoA), angle of departure (AoD) and Doppler frequency of multipath signals, is a critical task in perceptive mobile networks.

Although existing studies demonstrates the feasibility and potential of JCAS, most of them consider general signal formats, such as simple single carrier and multicarrier modulation [3], and is limited to point-to-point links such as millimeter wave radio for vehicular networks [4]. In [5], preliminary work on using orthogonal frequency-division multiplexing (OFDM) signal for sensing was reported. In [6], sparse array optimization was studied for multiple-input multiple-output (MIMO) JCAS systems. In [7], the multiple access performance bound is derived for a multiple antenna JCAS system. In [8], mutual information for an OFDM JCAS system is studied, and power allocation for subcarriers is investigated based on maximizing the weighted sum of the mutual information for radar and communications. However, there is only very limited work directly using modern mobile communication signals and

\footnotetext{
${ }^{1}$ This work is partially financially supported by China Academy of Telecommunications Technology (CATT), China.
}

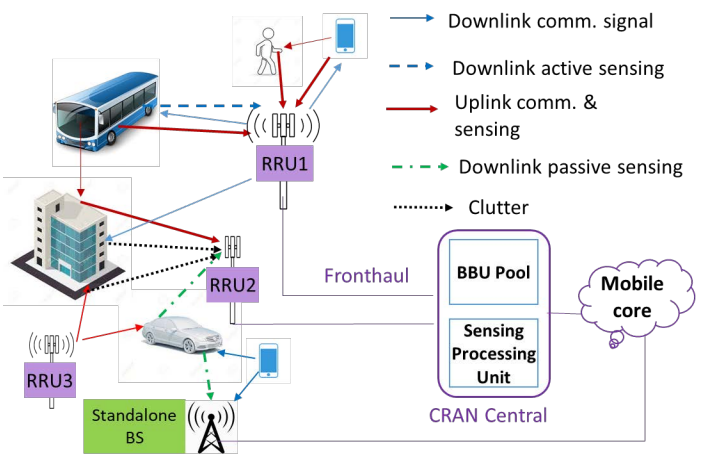

Fig. 1: Proposed perceptive mobile network with 5G CRAN.

networks in JCAS systems, involving orthogonal frequency division multiple access (OFDMA) and multi-user MIMO (aka spatial frequency division multiple access, SDMA). Such signal structure makes most existing sensing parameter estimation techniques not directly applicable. For example, active radar sensing technologies mainly deal with optimized or unmodulated transmitted signals [9], [10]; most passive sensing techniques consider simple single carrier and OFDM signals [11], [12]. In addition, conventional spectrum analysis techniques such as MUSIC [3] and ESPRIT [5] require continuous observations, which are not always available here. In turns, how JCAS can actually be realized at a system level in the mobile network, specifically, how radar sensing can be done based on communication signals using multiuser-MIMO and OFDMA technologies, is a fundamental and challenging problem. We will show that compressive sensing (CS) is an excellent candidate technology for this problem, after proper signal formulation.

In this paper, referring to the 5G NR standard, we study onedimension (1D) to 3D CS algorithms for estimating sensing parameters in perceptive mobile networks. These CS algorithms are developed from existing ones to make them capable of estimating all the sensing parameters. We consider both downlink and uplink sensing, to be consistent with downlink and uplink communications. The communication signals used for sensing are the OFDM-type demodulation reference signals (DMRS) in the 5G specification [13]. We use both 5G- 
compatible channels recommended by $3 \mathrm{GPP}$ and our own generated cluster channel model which has a better control for radio propagation for sensing purpose. We compare these CS algorithms, and demonstrate their respective advantages and disadvantages, under various channel conditions and system setup.

This rest of the paper is divided into three parts. The first part provides $5 \mathrm{G}$ usable signal and channel description. The second part describes various sensing parameter estimation algorithms including 1D, 2D, and 3D in details. In the third part, we discuss simulation results and outline a comparative study from these results obtained from $5 \mathrm{G}$ reference signals.

\section{Signal And Channel Models}

We consider 5G-compatible signals with OFDMA and SDMA (or multi-user MIMO, MU-MIMO) modulations. In a typical setup, there are 4 SDMA users, each with a single antenna, and a BS with a 16 antenna uniform linear array. The signal bandwidth is assumed to be $100 \mathrm{MHz}$. DMRS [13] is used as a primary signal for sensing. Propagation channels are generated based on clustered channel models of two forms. The first one is developed by us and named as Cluster$C h l$, and the second one is the QuaDRiGa channel model [14], recommended by $3 \mathrm{GPP}$ for modelling communication channels in LTE and $5 \mathrm{G}$ systems.

\section{A. DMRS Signal Generation}

DMRS signal is generated according to the Gold sequence as defined in [13] of 3GPP TS 38.211, both for Physical Downlink Shared Channel-PDSCH and Physical Uplink Shared Channel-PUSCH. The generated physical resourceblock (PRB) indicates DMRS to a 3-D grid comprising a 14-symbol slot for the full carriers across the DMRS layers or ports. The values and indices of DMRS signals are both known to the BS, and are used as prior when doing sensing from received signals. Here, interleaved DMRS subcarriers of PDSCH are used in downlink sensing, while groups of noninterleaved DMRS subcarriers of PUSCH are used in uplink sensing.

\section{B. Channel Modelling}

Random continuous values are used for delay, Doppler shift, AoAs and AoDs as actual sensing parameters. The multipaths of the channels are generated in clusters, indicating reflections coming from scattering obstacles. We generate approximate scatters for simulating sensing by using both the Cluster-Chl and QuaDRiGa model.

Consider a narrowband antenna array model [1], [2]. The array response vector of a size- $M$ array with $\theta$ of either AoD or AoA is,

$$
\mathbf{a}(M, \theta)=\left[1, e^{j \pi \sin (\theta)}, \cdots, e^{j \pi(M-1) \sin (\theta)}\right]^{H},
$$

For $M_{1}$ transmitting and $M_{2}$ receiving antennas, the $M_{2} \times$ $M_{1}$ time-domain baseband channel impulse response (CIR) matrix at time $t^{\prime}$ can be represented as

$$
\tilde{\mathbf{H}}\left(t^{\prime}\right)=\sum_{\ell=1}^{L} b_{\ell} \delta\left(t^{\prime}-\tau_{\ell}\right) e^{j 2 \pi f_{D, \ell} t^{\prime}} \mathbf{a}\left(M_{2}, \phi_{\ell}\right) \mathbf{a}^{T}\left(M_{1}, \theta_{\ell}\right)
$$

where for the $\ell$-th out of a total of $L$ multipath signals, $\theta_{\ell}$ and $\phi_{\ell}$ denote the AoD and AoA, respectively, $b_{\ell}, \tau_{\ell}$ and $f_{D, \ell}$ are the amplitude, propagation delay, and Doppler frequency, respectively.

1) Cluster-Chl Channel Model: In Cluster-Chl, we can have a flexible and accurate control on all the channel parameters including AoA, AoD, Doppler shift, delay, and amplitude, and then verify with sensing results. The multipath channels of Cluster-Chl are randomly generated in clusters following a complex Gaussian distribution to generate sensing parameters for moving objects around the mobile network node, which mimics the ray tracing model.

For the channels used in this paper, we generate 3 clusters with delay centered at 29,39 and $49 \mu \mathrm{s}$, corresponding to a distance of 87, 117 and 147 meters, with AoA center randomly generated between -120 to 120 degrees, and moving speed randomly generated between 0 to $40 \mathrm{~m} / \mathrm{s}$. In each clusters, as like we did in [1], multipath signals for each RRU/MS are generated randomly by mimicking reflected/scattered signals from objects. In each cluster, the multipath is generated following a uniform distribution of $[5,10]$ for the total number, $[0,28]$ degrees for direction span, $[0,0.05] \mu$ s for delay (corresponding to $[0,15] \mathrm{m}$ for distance). We use a pathloss model with pathloss factor 40 for downlink and 20 for uplink sensing. The transmission power of the RRU and MS is 30 $\mathrm{dBm}$ and $25 \mathrm{dBm}$ respectively. The total thermal noise in the receiver is $-174+10 \log \left(10^{8}\right)=-94 \mathrm{dBm}$.

2) QuaDRiGa Channel Simulator: QuaDRiGa is a spatial geometry based 3D MIMO channel generator [14], originated from the WINNER series models and supports rich cluster multipath scenarios specified by the 3GPP-3D cluster-based models mentioned in $T R 36.873$ and TR36.901 [14]. We generate QuaDRiGa channels equivalent to moving scatters by simulating moving transmitters and receivers from open source simulator for sensing demonstration. Non-line of sight channels are simulated. One problem with the QuaDRiGa model is that scatters can not be accurately placed and configured. The other problem is that the Doppler frequencies for each multipath is not explicitly provided and hence we cannot verify the accuracy of estimates.

\section{Sensing Parameter Estimation}

We develop and test 1D, 2D and 3D CS algorithms for sensing parameter estimation using the received signals. These algorithms are extended from the 1D-CS algorithms [15], 2D Kronecker CS [16], and 3D N-way Tensor tool [17]. We test these algorithms utilizing Cluster-Chl in downlink sensing and QuaDRiGa model in uplink sensing. We also compare our 1D 
to $3 \mathrm{D}$ results with the cases when the occupied PRB is small in uplink and with the results from 2D discrete Fourier transform (DFT).

\section{A. Signal Model for Sensing}

The received radar signal data is based on $3 \mathrm{D}$ observation samples, comprising of those from multiple receiving antennas, multiple DMRS subcarriers and multiple DMRS signals over time. The modulated data symbols can be removed from the received DMRS signals by applying equalization, which will be a simple one-tap multiplication if no two users are sharing the same subcarrier. In this paper, we consider the case that only each subcarrier is used by only one user in DMRS signals. After the equalization, the processed received signal at the $n$-th subcarrier and the $t$-th DMRS signal can be represented as

$$
\begin{aligned}
& \mathbf{Y}_{n, t}= \sum_{\ell=1}^{L} b_{\ell} e^{-j 2 \pi n \tau_{\ell} f_{0}} e^{j 2 \pi t f_{D, \ell} T_{s}} . \\
& \mathbf{a}\left(M_{2}, \phi_{\ell}\right) \mathbf{a}^{T}\left(M_{1}, \theta_{\ell}\right)+\mathbf{z}_{n, t}, \\
&=\mathbf{A}_{r x} \mathbf{C}_{n} \mathbf{D}_{t} \mathbf{A}_{t x}^{T}+\mathbf{z}_{n, t},
\end{aligned}
$$

where the $\ell$-th column in $\mathbf{A}_{r x}$ and $\mathbf{A}_{t x}^{T}$ are $\mathbf{a}\left(M_{2}, \phi_{\ell}\right)$ and $\mathbf{a}^{T}\left(M_{1}, \theta_{\ell}\right)$, respectively; if there is no two multipath having the same delay values, $\mathbf{D}_{t}$ and $\mathbf{C}_{n}$ are diagonal matrices with the $\ell$-th diagonal element being $b_{\ell} e^{j 2 \pi t f_{D, \ell} T_{s}}$ and $e^{-j 2 \pi n \tau_{\ell} f_{0}}$, respectively, otherwise there will be non-zero values in other entries; and $\mathbf{Z}_{n, t}$ is the noise matrix. When each user only has one transmitting antenna, $\mathbf{A}_{t x}^{T}$ becomes a all-one column vector, and $\mathbf{Y}_{n, t}$ and $\mathbf{Z}_{n, t}$ become column vectors too. The task for sensing parameter estimation is to estimate $\left\{\tau_{\ell}, f_{D, \ell}, \phi_{\ell}, \theta_{\ell}, b_{\ell}\right\}, \ell \in[1, L]$ from the received signals. In this paper, we only consider the estimation of the first three parameters.

\section{B. Estimation of Sensing Parameters}

Since the signals are relatively independent in the three domains of delay, AoA and Doppler, they can be formulated in a high-dimension (3D here) vector Kronecker product form. Therefore, we can apply 1D to 3D CS techniques to estimate these sensing parameters. In a typical system, we can get sufficient number of observations for the delay (linked to number of subcarriers), intermediate AoA observations (linked to number of antennas) and a limited number of samples in the Doppler domain (linked to DMRS signals over a portion of channel coherent period). The Doppler frequency is typically very small in a perceptive mobile network and the accumulated phase shift usable is also small due to the limited period of channel coherent time. This makes it inaccurate for estimating Doppler using CS algorithms. Next, we briefly review each of the three sensing algorithms based on received signal in (4).

1) $1 D$ Compressive Sensing: We assume that there is only one multipath signal within each quantized delay bin for each cluster in the 1D CS based algorithm. By stacking the signals in (4) from all available subcarriers to one matrix, we can get

$$
\mathbf{Y}_{t}=\mathbf{W} \underbrace{\mathbf{D}_{t} \mathbf{A}_{r x}^{T}}_{\mathbf{G}_{t}}+\mathbf{Z}_{t},
$$

where the $\ell$-th column of $\mathbf{W}$ is $\left\{e^{-j 2 \pi n \tau_{q}, \ell} f_{0}\right\}$. We can then treat (5) as an on-grid multi-measurement vector (MMV) CS problem and use algorithms such as 1D sparse Bayesian CS to get the estimate for $\mathbf{G}_{t}$. The dictionary $\boldsymbol{\Psi}_{1}$ is a partial DFT delay matrix, approximating $\mathbf{W}$. Once the delays and $\mathbf{G}_{t}$ are estimated, we can get the AoA estimates through calculating the cross-correlation between columns from $\mathbf{G}_{t}$ on the indexes obtained from given threshold as below,

$$
\phi_{\ell} \approx \frac{1}{\pi} \angle\left(\sum_{p=1}^{M-1}\left(\left(\mathbf{G}_{t}\right)_{\cdot, p}\right)^{*}\left(\mathbf{G}_{t}\right)_{\cdot, p+1}\right),
$$

where $\left(\mathbf{G}_{t}\right)_{\cdot, p}$ denote the $p$-th column $\mathbf{G}_{t}$.

The Doppler frequency $f_{D, \ell}$ can be estimated across multiple DMRS signals, based on the cross-correlation of $\left(\mathbf{G}_{t}\right)_{\ell, \text {, }}$,

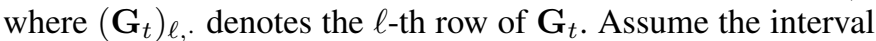
between every two estimates of $\mathbf{G}_{t}$ and $\mathbf{G}_{t+1}$ is uniform and be $T_{s}$ for any $t$, which can be relaxed easily. Let $N_{d}$ be the total OFDM blocks used for estimating the Doppler frequency. Then,

$$
f_{D, \ell} \approx \frac{1}{2 \pi T_{s}} \angle\left(\sum_{t=1}^{N_{d}-1}\left(\left(\mathbf{G}_{t}\right)_{\ell, \cdot}\right)\left(\left(\mathbf{G}_{t+1}\right)_{\ell, \cdot}\right)^{*}\right) .
$$

The main advantage of the $1 \mathrm{D}$ algorithm is that it can accurately estimate all the parameters when each multipath is well separated in delay. Its complexity is also relatively low. It is mostly suitable for systems with a large number of subcarriers, but small number of antennas for AoA estimation and packets for Doppler estimation.

2) 2D Kronecker Compressive Sensing: 2D Kronecker CS can obtain direct estimation for any two parameters out of delay, AoA and Doppler. Since we can have sufficient measurements in the delay and AoA domain, the 2D CS algorithm can provide good estimates for both delay and AoA directly from $\mathbf{Y}_{t}$ of (5) using each DMRS signal of 2D observations. We construct two dictionaries for delay and AoA, $\Psi_{1}$ and $\boldsymbol{\Psi}_{2}$, being two partial overcomplete DFT matrices, approximating $\mathbf{W}$ and $\mathbf{A}_{r x}$, respectively. Interpolated overcomplete dictionaries are used to improve resolution, given that the signal to noise power ratio (SNR) is sufficiently large, at an increased computational complexity. We can then obtain estimates $\hat{\mathbf{D}}_{t}$ for the expanded matrix for $\mathbf{D}_{t}$ that corresponds to the two overcomplete dictionaries, using any 2D Kronecker CS algorithm, such as the 2D-OMP algorithm [16].

Note that 2D CS algorithm here can identify any pair of $\{$ delay, AoA $\}$ with at least one different values. So $\hat{\mathbf{D}}_{t}$ will not be a diagonal matrix anymore if one variable in the pair has two identical quantized values.

After getting the estimate $\hat{\mathbf{D}}_{t}$, we use a threshold to filter out very small estimates which are likely caused by noise. 
We can then get the delay and AoA estimates according to the indexes of the non-zero values in $\hat{\mathbf{D}}_{t}$, corresponding to respective columns in the two dictionaries.

The Doppler shift is estimated via calculating the angle of the cross-correlation values between the non-zero values of $\hat{\mathbf{D}}_{t}$ obtained over two DMRS signals. This can be represented as

$$
f_{D, q, \ell} \approx \frac{1}{2 \pi T_{s}} \angle\left(\hat{\mathbf{D}}_{t} \hat{\mathbf{D}}_{t+1}^{*}\right)_{\ell, \ell} .
$$

Averaging can be taken over the correlation obtained from multiple DMRSs before computing the angle, to improve the accuracy of the estimates.

2D CS algorithm can achieve improved estimation accuracy when there are multipath with repeated values in any one domain, at the cost of increased complexity.

3) 3D Tensor Compressive Sensing: The Tensor-OMP CS algorithm directly estimate parameters in a 3D domain, combining measurements $\mathbf{Y}_{t}$ over multiple DMRS signals. Three dictionaries, $\boldsymbol{\Psi}_{1}, \boldsymbol{\Psi}_{2}$, and Doppler dictionary matrix, $\boldsymbol{\Psi}_{3}$, are utilized. Since the accumulated Doppler shift is small over the coherent time period, we have to use a portion of highly overcomplete DFT matrix as $\boldsymbol{\Psi}_{3}$.

Absolute values of the estimated sparse coefficients provides the amplitude values of multipaths. After applying a threshold, each of indexes in the three dimensions corresponding to nonzero estimates provides estimated values for delay, AoA and Doppler shift.

3D CS algorithm has the highlight complexity, and in principle provides the strongest capability in resolving multipath with repeated parameter values. However, due to the small value of Doppler shift, we will see that it does not work as well as $1 \mathrm{D}$ and $2 \mathrm{D}$ algorithms.

\section{Simulation Results}

Next, we present simulation results for 1D to 3D CS using channels with continuous-value (off-grid) sensing parameters. We also show the results that obtained by directly applying 2D-FFT over delay-AoA and delay-Doppler domains for comparison, when all subcarriers are used. Of course, most of the time, not all subcarriers are available for sensing. Estimated values (typically shown in blue star) are placed with actual ones (shown in red circle) to verify the accuracy of estimation. Fig. 2 shows an exemplified CIR for QuaDRiGa channels consisting of rich multipaths with 4 to 5 clusters.

\section{A. Downlink Sensing}

In downlink sensing, we use DMRS subcarrier configuration type-1 slot wise, with every alternating subcarriers as interleaved selection from a total of 252 subcarriers. Sub-interval is 2 for type-1, so, in total 126 subcarrier indices (e.g. of layer 4) are used as DMRS subcarriers.

The simulation results for downlink sensing with ClusterChl model are presented in Fig. 3 for 1D to 3D. For 1-D CS, AoA-Distance and Speed-Distance result indicates that the estimated points are well matched with three clusters with few extra points due to residual in threshold setting. Off-grid
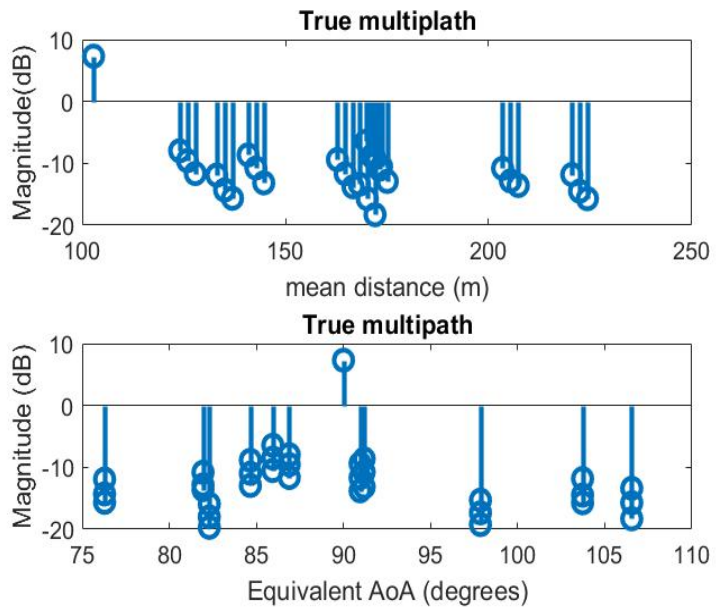

Fig. 2: CIR for QuaGRiGa channel.

estimation causes some missed detection. In 2D CS, AoADistance and Speed-Distance results gives few mismatched points for both AoA and speed. In 2D, there is an important observation that interleaved subcarriers in this case actually cause ambiguity in estimation. 3D estimation results is not as good as 1D and 2D CS algorithms as in 3D estimation the interleaved subcarriers cause near-singular matrix.

The ambiguity is caused by non-consecutive, but regularly spaced samples, for example, usage of comb/interleaved subcarriers [18]. In this case, the actual value can be one of the multiple integral times of a basic estimate. The simplest way is to break such regularly spaced samples, for example, we can randomly select samples from the total available ones such that the indexes of these samples are not regular. This of course reduces the samples used for estimation and may degrade the estimation performance particularly when the number of samples are small. Therefore, while using DMRS, alternative methods can be based on exploiting other information to assist the selection of the right estimate, for example, the magnitude, or an integration of coarse and fine estimation methods. This is in fact one of our future work to focus on how to solve ambiguity problem that may be present in all domains, but particularly for delay.

\section{B. Uplink Sensing}

In uplink sensing, only partial DMRS subcarriers are used. The details of obtained results from non-line-of-sight (NLOS) QuaDRiGa channel model are given here. We use DMRS subcarrier configuration type-2 non-slot wise here, which indicates several groups of subcarriers are selected from a total of 252 subcarriers. Sub-interval is 3 for type-2, so, in total 84 subcarrier indices (of layer 4) are used as DMRS subcarriers. We use QuaDRiGa model where clusters are generated with continuous delay and AoA values for multipaths. Since the QuaDRiGa model unable to provide actual Doppler shifts, the estimates given here in all uplink sensing for speed is relative only. 

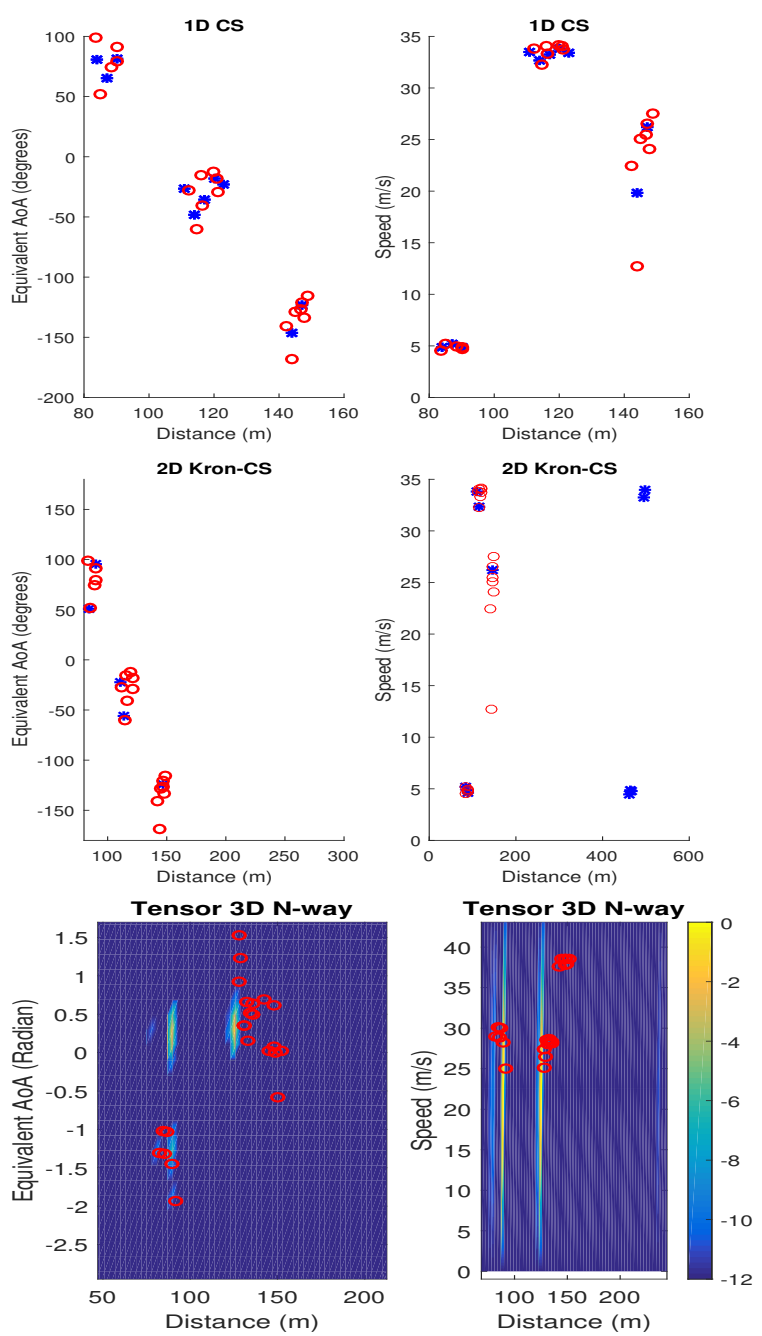

Fig. 3: Observations in Cluster-Chl for downlink sensing.

1) Uplink Sensing with QuaDRiGa NLOS: Fig. 4 provides simulation results for uplink sensing with QuaDRiGa NLOS for $1 \mathrm{D}$ to 3D. In 1-D CS, AoA-distance results for the estimated points fairly match with several clusters with few extra points due to residual in threshold setting and off-grid error.

2D CS AOA-distance results indicates that, estimated values are in closer vicinity with actual clustered multipath values (AoA) in comparison with 1D. Indeed, higher dimensional sensing algorithm like the 2D kron CS eventually provide better performance when there is enough measurements because it can directly identify two parameters only if one is different between any multipath channels. In both figures for 3D NOMP, only estimated multipath channels with power within $-15 d B$ of the maximum are shown. In $3 \mathrm{D}$, estimated values for AoA are coarser, but remain within the close neighbouring of actual values.

2) 2D FFT results for uplink Sensing: 2D-DFT simulation results are presented in Fig. 5 for QuaDRiGa channels. It can be observed that 2D-DFT provides reliable coarse estimates for
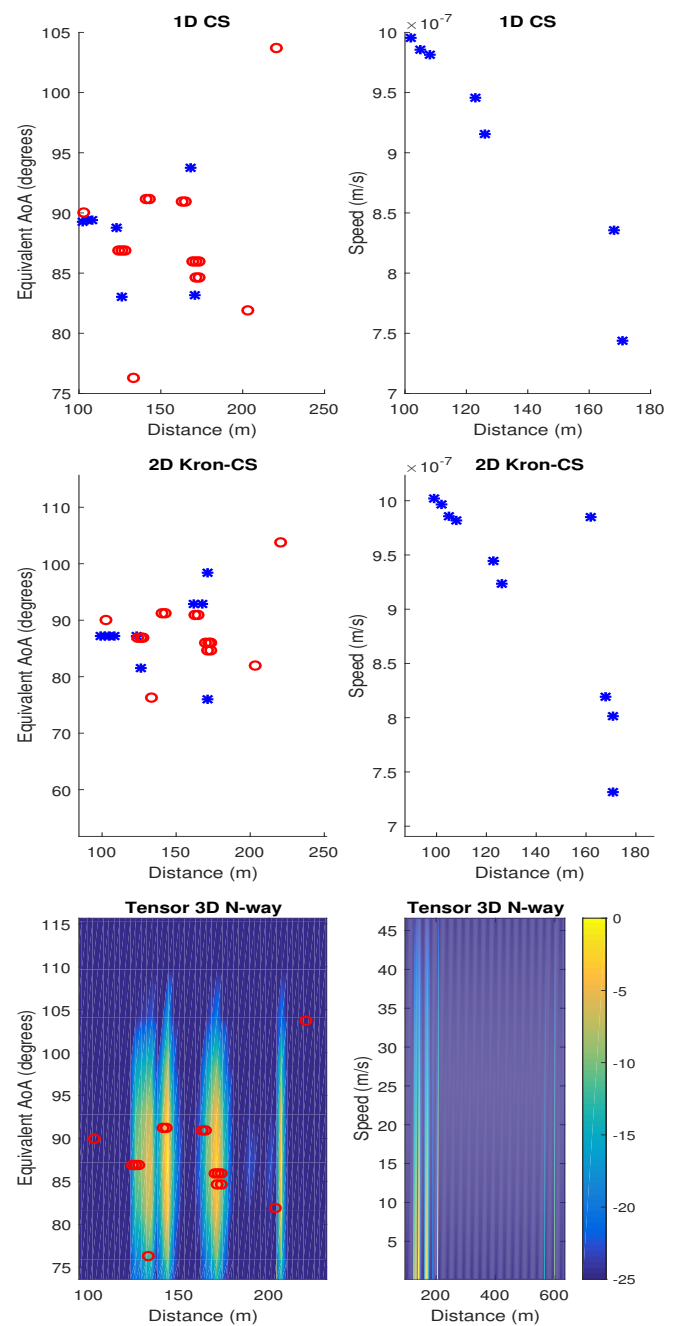

Fig. 4: Observations in uplink sensing: QuaDRiGa NLOS channel.
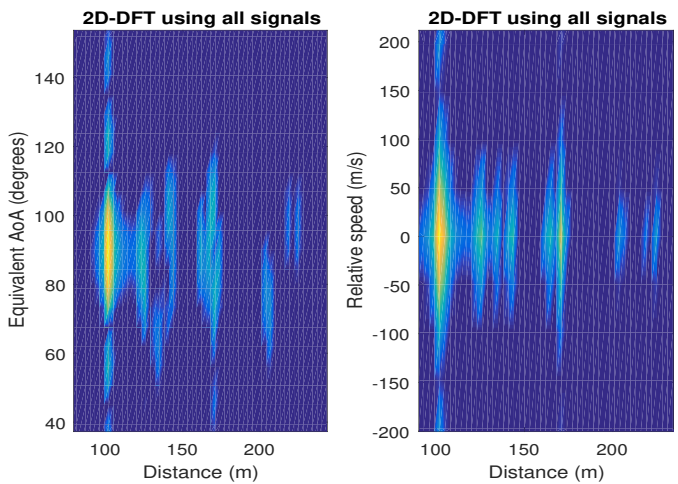

Fig. 5: 2-D DFT for uplink sensing.

uplink sensing, but the resolution is very low in comparison with all results of $1 \mathrm{D}$ to $3 \mathrm{D}$ in Fig. 4 . It is noted that here the results are obtained by using all subcarriers. Such a 2D-FFT method only works when either all or interleaved subcarriers are available. 

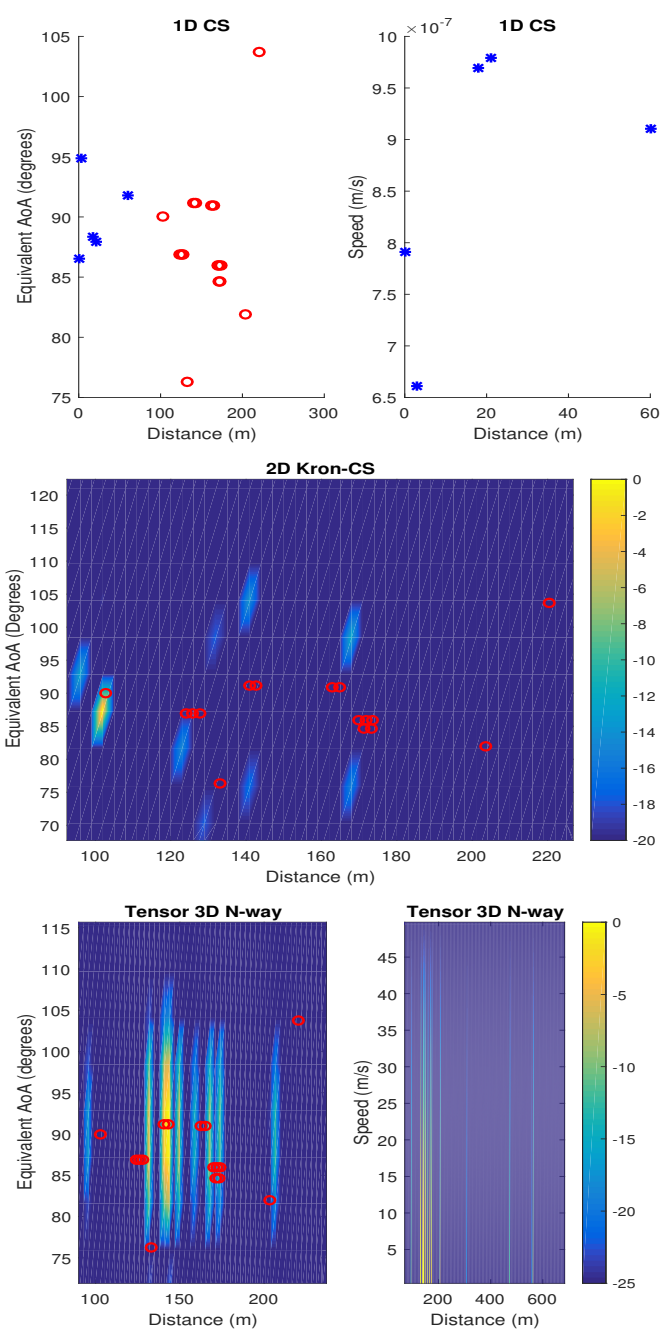

Fig. 6: Uplink sensing with small PRB in 5G QuaDRiGa NLOS.

\section{Sensing Using Small Number of PRB}

Mainly in uplink direction, allocated PRB could be limited by configuration. Therefore, we can only get small amount of observations for the delay estimation, which could be even less than the number of multipath. We test sensing with such limited number of resources for QuaDRiGa NLOS channel here. Assume that only 28 DMRS subcarriers (7 PRB) are used in simulations for uplink sensing. Fig. 6 presents uplink sensing for small PRB with QuaDRiGa NLOS for 1D to 3D. In $1 \mathrm{D}$ CS, resolution ambiguity problem remains in the delay domain. However, the shape still well maintained. Reasonable estimation accuracy is found in AoA by 2D. Coarse estimation in both AoA and speed is obtained by 3D. Further approaches of increasing this accuracy in radio sensing from limited observations, especially in clustered multipath environment will be studied in our future research, for example, by designing better dictionaries and using filtering techniques.

\section{CONClusion}

We presented three preliminary sensing algorithms using 1D, 2D and 3D compressive sensing algorithms, and provided simulation results, using channels generated from both our own cluster model and 5G QuaDRiGa channel model. These results indicate that reasonable sensing performance can be achieved, and demonstrate respective advantages and disadvantages of these algorithms. Our work also disclosed some interesting research problems to work on as future works, such as the ambiguity problem due to interleaved subcarriers and reduced resolution in 3D CS algorithms.

\section{REFERENCES}

[1] J. A. Zhang, A. Cantoni, X. Huang, Y. J. Guo, and R. W. Heath, "Framework for an innovative perceptive mobile network using joint communication and sensing," in 2017 IEEE Vehicular Technology Conference (VTC Spring), June 2017, pp. 1-5.

[2] J. A. Zhang, X. Huang, Y. J. Guo, and M. L. Rahman, "Signal stripping based sensing parameter estimation in perceptive mobile networks," in 2017 IEEE-APS Topical Conference on Antennas and Propagation in Wireless Communications (APWC), Sept 2017, pp. 67-70.

[3] C. Sturm and W. Wiesbeck, "Waveform Design and Signal Processing Aspects for Fusion of Wireless Communications and Radar Sensing," in Proceedings of the IEEE, vol. 99, no. 7, pp. 1236-1259, July 2011

[4] P. Kumari, J. Choi, N. Gonzlez-Prelcic and R. W. Heath, "IEEE 802.11ad-Based Radar: An Approach to Joint Vehicular Communication-Radar System," in IEEE Transactions on Vehicular Technology, vol. 67, no. 4, pp. 3012-3027, April 2018.

[5] M. Braun, "OFDM radar algorithms in mobile communication networks," PhD thesis, Institut fur Nachrichtentechnik des Karlsruher Instituts fur Technologie, Karlsruhe, 2014.

[6] X. Wang, A. Hassanien, and M. Amin, "Dual-function MIMO Radar communications system design via sparse array optimization," IEEE Transactions on Aerospace and Electronic Systems, vol. PP, pp. 1-1, 082018.

[7] Y. Rong, A. R. Chiriyath, and D. W. Bliss, "Multiple-antenna multipleaccess joint radar and communications systems performance bounds," in 2017 51st Asilomar Conference on Signals, Systems, and Computers, Oct 2017, pp. 1296-1300.

[8] Y. Liu, G. Liao, J. Xu, Z. Yang, and Y. Zhang, "Adaptive OFDM integrated radar and communications waveform design based on information theory," IEEE Communications Letters, vol. 21, no. 10, pp. 2174-2177, Oct 2017.

[9] A. M. Haimovich, R. S. Blum and L. J. Cimini, "MIMO Radar with Widely Separated Antennas," in IEEE Signal Processing Magazine, vol. 25, no. 1, pp. 116-129, 2008.

[10] J. Liang and Q. Liang, "Design and Analysis of Distributed Radar Sensor Networks," in IEEE Transactions on Parallel and Distributed Systems, vol. 22, no. 11, pp. 1926-1933, Nov. 2011.

[11] D. E. Hack, L. K. Patton, B. Himed and M. A. Saville, "Detection in Passive MIMO Radar Networks," in IEEE Transactions on Signal Processing, vol. 62, no. 11, pp. 2999-3012, June1, 2014.

[12] L. Zheng and X. Wang, "Super-Resolution Delay-Doppler Estimation for OFDM Passive Radar," in IEEE Transactions on Signal Processing, vol. 65, no. 9, pp. 2197-2210, 1 May1, 2017.

[13] 3GPP TS 38.211, Physical channels and modulation, V15.2.0, July 2018.

[14] 3GPP TR 38.913, Study on scenarios and requirements for next generation access technologies, V15.0.0, June 2018.

[15] S. Ji, Y. Xue, and L. Carin, "Bayesian compressive sensing," in IEEE Transactions on Signal Processing, vol. 56, no. 6, pp. 2346-2356, June 2008.

[16] M. F. Duarte and R. G. Baraniuk, "Kronecker compressive sensing," in IEEE Transactions on Image Processing, vol. 21, no. 2, pp. 494-504, Feb 2012

[17] C. F. Caiafa and A. Cichocki, "Computing sparse representations of multidimensional signals using Kronecker bases," in Neural Computation, vol. 25, no. 1, pp. 186-220, Jan 2013.

[18] G. Hakobyan and B. Yang, "A novel OFDM-MIMO radar with nonequidistant subcarrier interleaving and compressed sensing," in 2016 17th International Radar Symposium (IRS), Krakow, 2016, pp. 1-5. 